\title{
Using a Wireless Visual Sensor Network to Harmonically Navigate Multiple Low-Cost Wheelchairs in an Indoor Environment
}

\author{
Feng Tian ${ }^{\mathrm{a}, \mathrm{b}}$, Kuo-Ming Chao ${ }^{\mathrm{c}}$, Zuren Feng ${ }^{\mathrm{b}}$, Keyi Xing ${ }^{\mathrm{b}}$, Nazaraf Shah ${ }^{\mathrm{c}}$ \\ ${ }^{a}$ MOE Key Lab for Intelligent Networks and Network Security, Xi'an Jiaotong \\ University, Xi'an, China \\ ${ }^{b}$ Systems Engineering Institute, Xi'an Jiaotong University, Xi'an, China \\ ${ }^{c}$ Faculty of Engineering and Computing, Coventry University, Coventry, UK
}

\begin{abstract}
Harmonic navigation of multiple low-cost robotic wheelchairs in a topology of wireless sensor nodes that are deployed in a dynamic and crowded indoor environment is a Non-deterministic Polynomial-time hard (NP-hard) problem. To address this problem, we propose a distributed multi-wheelchair global harmonic navigation algorithm. The distinguishing features of the proposed navigation algorithm are global search and local conflict resolution abilities. In the proposed algorithm, a travel time prediction method adopts a penalty for potential conflicts based on wheelchairs' priority, velocity and distance between the nodes. Moreover, three harmonic rules are proposed for: 1) giving the highest priority to humans, 2) giving the highest priority to wheelchairs, 3) giving flexible priority to wheelchairs. Through extensive quantitative simulations, we explore the performance of wheelchairs in various floor plan topologies and different values for the system parameters, and demonstrate that the properties of crowded indoor environments have important influence on the performance of global navigation, such as service time. The third harmonic rule establishes the trade-off between the performance of humans and robotic wheelchairs. At the same time, physical prototype wheelchairs are implemented and they verify the proposed global harmonic navigation algorithm. Some suggestions for robotic wheelchair designers,
\end{abstract}

Email addresses: fengtian@mail.xjtu.edu.cn (Feng Tian), csx240@coventry.ac.uk ( Kuo-Ming Chao), fzr9910@mail.xjtu.edu.cn (Zuren Feng), kyxing@mail.xjtu.edu.cn (Keyi Xing), aa0699@coventry.ac.uk (Nazaraf Shah)

Preprint submitted to Journal of Network and Computer Applications October 10, 2015

(C) 2015. This manuscript version is made available under the Elsevier user license

http://www.elsevier.com/open-access/userlicense/1.0/ 
building architects and building owners are provided based on the conclusion of the experimental results.

Keywords: harmonic navigation, low-cost wheelchair, multiple wheelchair navigation, wireless visual sensor network, indoor environment

\section{Introduction}

Robotic wheelchairs are a type of service robot for indoor applications, such as health care, and will co-exist in the human environment in near future. Autonomous and harmonic navigation is a remarkable feature of robotic wheelchairs. Many applications have focused on developing an integrated wheelchair with a smart 'brain' that can perceive and understand its environment, and respond to changes in that environment (Yuan, 2009; Simpson, 2005). These applications especially emphasize on giving highest priorities to humans (Okorn et al., 2010; Sisbot et al., 2007; Lam et al., 2011) in order to ensure harmonious human-robot coexistence.

Recently, there is a growing research trend in mutual beneficial collaboration (Batalin et al., 2004; Payton et al., 2001; Li and Shen, 2011; Wu et al., 2014) between mobile robots and static sensor networks, as it is believed that in the future, there will be a potential and need for such practical applications (Batalin et al., 2004) in the area of search and rescue and disable assistance. In such applications, neither network nodes nor mobile robots need to know their positions or build any kind of map (O'Hara et al., 2008), and mobile robots are guided autonomously between different locations by the navigation network. These applications also aimed at building an intelligent environment to support the robot navigation, and they preferably find shortest possible paths while avoiding dangers in the environment ( $\mathrm{Li}$ and Rus, 2005), and collisions with static obstacles (O'Hara et al., 2008), and other mobile objects. Motivated by the characteristic of this kind of network, we have described a solution (Jiang et al., 2011; Tian et al., 2007), in which wireless visual sensor nodes (WVSnode) are distributed in an intelligent environment to support navigation of a robotic wheelchair. The distributed sensors and associated distributed information can release massive robot intelligence in its inhibited environment, which consists of a wireless visual sensor network (WVSN) that detects robots, collects environment information and activates robot behaviors in order to respond to the emergent events and help the robots to achieve their goals. But how to use this kind of intelligent environ- 
ment to harmonically navigate multiple low-cost wheelchairs in an indoor environment is not considered in extant research. This is a challenging problem. Firstly, for current harmonic rules (i.e. designing robotics to serve humans and giving the highest priorities to humans (Okorn et al., 2010; Sisbot et al., 2007; Lam et al., 2011)), some researchers have realized that the situation is quite different for robotic wheelchairs in the sense that wheelchairs may have the highest priorities to move sometime when humans are aware of their existence (Murakami et al., 2001; Kuno et al., 2003). Secondly, current navigation algorithms are not suitable for an indoor environment that is characterized by narrow or crowded aisles. For analyzing this environment, such a class of navigation networks can be considered as a weighted graph, for example as shown in Figure 1. In which, a shortest path between $v_{1}$ and $v_{6}$ may be not an optimal solution for two robots running in a opposite direction, when sum of the costs involved in multiple robotic wheelchair travel is considered. Furthermore, a task of planning multi-wheelchair navigation in a crowded and dynamic environment by applying our proposed network can be characterized as a NP-hard problem (Toth and Vigo, 2001). The detailed discussion on Figure 1 is described in Section 2. Thirdly, current research on harmonic navigation focuses on designing local navigation algorithms. For instance, Sisbot et al. (2007) developed a method of determining how a robot may approach a human by considering safety criteria, visibility criteria, and hidden zones. This kind of research has improved the behavior control method or mechanism of a single wheelchair, but it does not focus on global navigation planner. Some researchers have investigated the harmonic global navigation of multiple wheelchairs. For an instance, Guzzi et al. (2013) implemented a fully distributed algorithm for robot local navigation, as well as a heuristics strategy for mutual avoidance of humans. They also found that the emergent collective behaviors are similar to those observed in human crowds. However, this research did not consider a crowded indoor environment and node topology-based navigation. At the same time, we believe that the wheelchair users have an equal right to access the paths and the quality of serving time, especially when they face emergent situations with time constraints, such as meeting a doctor. This means that the serving time should be considered in the context of global navigation. Moreover, because the distribution of our designed sensor network, the dynamic environment, and limited measuring range of sensors installed on a robotic wheelchair, the central scheduler or planner that persists in global optimal solution is infeasible. In fact, in this kind of real-world environment, it is necessary to design 
more flexible distributed algorithms for multiple robotic wheelchair navigation to create a harmonic environment for human-robotics collaboration and explore different scenarios and their impact on the algorithms.

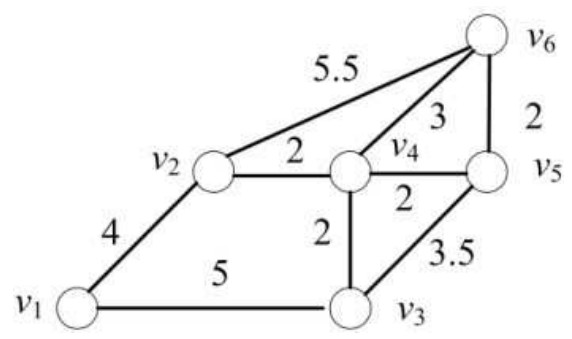

Figure 1: An example of a weighted graph

The contributions of this paper include (1) a multi-wheelchair navigation algorithm based on a WVSN for narrow space scenarios in indoor applications, which is featured as global searching and local conflict resolution, (2) a travel time prediction method that adopts the penalty for potential conflicts based on wheelchair velocity and distance between nodes and various harmonic rules from a perspective of trade-off between travel performance of humans and robotic wheelchairs, and (3) performance evaluation of the proposed algorithm in various topologies of classical floor plans in indoor environments.

The remainder of this paper is organized as follows. Related work is reviewed in Section 2. An overview of our navigation network is described in Section 3. The distributed navigation algorithm with harmonic rules are described and evaluated using various topology simulation scenarios in Section 4. The detailed information of a prototype system based on wireless visual nodes and real-world experiments and their results are introduced in Section 5. Finally, the paper is concluded in Section 6.

\section{Related work}

Many researchers paid attention to intelligent wheelchair to build a central control unit, such as an agent running on a laptop/remote controller (Lu et al., 2006; Faria et al., 2014; Chen et al., 2013). Trieu et al. (2008) 
built an intelligent wheelchair that can detect obstacles in front of it using a laser range finder sensor, and produces a real-time map. They have considered the user's intentions via head-movement interface, accessible space of the environment and user safety in their control method. Del Castillo et al. (2006) adopted a sonar approach to detect obstacles for a vision based autonomous wheelchair.

Recently, Radio Frequency Identification (RFID) technology has been introduced into intelligent wheelchair systems, especially for marking global information label (Hamagami and Hirata, 2004; Matsumoto et al., 2006; Tao et al., 2009). Hamagami and Hirata (2004) implemented a software agent to control autonomous behaviors, such as cognising the safety and effectiveness of every move by observing a local real environment by range sensors and acquiring more global and accurate information, such as room number. Matsumoto et al. (2006) built a wheelchair with the ability to accurately determine its position and direction using internal sensors and external assistance from the surrounding environment, including Global Positioning System (GPS) and RFID. Its autonomous traveling controller is a control unit, a board computer and motor amplifiers. Tao et al. (2009) equipped their wheelchair with a main controller (via embedded board $1.5 \mathrm{G}$ ), where wheel encoders and a ultrasonic sensor are used for obstacle avoidance and a RFID reader is used for detecting the RFID tags on the floor or furniture for location.

With the development of wireless sensor network (WSN) technology, some researchers on robotics have tried to explore a new way of combining WSN and robotics to better serve all kinds of practical applications (Akyildiz et al., 2007; Chen et al., 2013). Some research efforts emphasized on platform implementation and distributed navigation algorithms. O'Hara et al. (2008) presented a hardware platform, the GNATs, to aid in path planning, in which the nodes are equipped with four infrared (IR) emitters and four IR receivers. In the path finding stage a node broadcasts the message between nodes in the similar way to distributed Bell-ford algorithm. Simpson (2005) adopted an approach of using embedded nodes to create a 'navigation field', in which mobile robots can find their way around. In this author's approach, transition probabilities between nodes were estimated to compute a best direction to suggest to a mobile robot for moving between a source and a destination. $\mathrm{Li}$ and Rus (2005) used number of hops to evaluate the distance between sensors without relying on location information and relying on a reactive task in a sensor network for guiding the movement of a user equipped with a node that can talk to sensors across the field. Bhattacharya et al. (2006) 
have described a Roadmap Query based navigation method for outdoor and dynamic environment applications. Baeg et al. (2007) and Louloudi et al. (2010) introduced service oriented robots for smart home applications, but both of them do not provide the solution to the problem of coordinating multiple wheelchairs, especially in indoor applications.

Some researchers concentrated on collaboration of fully mobile, large scale and small size nodes (Wu et al., 2014). For example, Payton et al. (2001) introduced a system, named 'Pheromone Robotics', inspired by biology (swarm behavior) for large scale multi-robot control, in which every node is the same as a robot and called as mobile node. They have ability to move, communicate and make decision. The mobile nodes equipped with a wireless sensor form dynamic groups, share information and adopt wireless communication distance to evaluate the distance between them.

The aforementioned research strands developed wireless visual nodes and distributed navigation algorithms to support multiple robotic wheelchair navigation in an indoor environment while taking the environment constraints into consideration. In such applications distributed sensor nodes consist of a wireless visual sensor network that can detect robots, collect environment information, and activate robot behaviors through emergent events and goals, in order to release the massive robot intelligence in its living environment. In previous works (Jiang et al., 2011; Tian et al., 2007), we have described our solution to some basic problems of how to design a robot capable of knowing: 1) where am I going to? 2) Where am I? and 3) how do I get there?. Furthermore, our prior research introduced a major phase of navigation and described some simulation experiments that were carried out to evaluate some algorithms, such as, Random Selection Policy (RaSelP), RaSelP+Plan-Break, broad-first-search-like policy-based Plan-Forward without Punishment (BFSP+FWoutP), and broad-first-search-like policy-based Plan-Forward with Punishment (BFSP+Plan-FWP) (Tian and Feng, 2012), according to the indicators, such as energy consumption per node and the number of messages sent in the whole network.

Recently, the issue of harmonic movement of multiple robotic wheelchairs in a crowded environment has been brought into academic and robotic engineering field. Current research (Mihailidis et al., 2007; Kobavashi et al., 2013; Cao et al., 2009; Kuderer et al., 2013; Okorn et al., 2010; Sisbot et al., 2007; Lam et al., 2011; Guzzi et al., 2013) focuses on guiding a wheelchair robot to harmonically pass a crowded environment and give highest priority to humans. For example, Lam et al. (2011) proposed six harmonic rules for 
robots to follow in order to guarantee least disturbance during navigation in a human-robot environment. Hamagami and Hirata (2004) proposed a method for predicting human's behavior during movement and performing avoidance behavior by employing a social force model. All the harmonic rules designed for local navigation algorithms follow the objectives that serve humans and give the highest priorities to humans. Some researchers believe that this kind of situation is quite different for robotic wheelchairs in a sense that wheelchairs may have the highest priorities to move when humans are aware of their existence (Tomari et al., 2014). Face-to-face direction cases based on the observation of collision avoidance patterns in hospital corridor were proposed in wheelchair navigation in human-shared environments (Murakami et al., 2001; Kuno et al., 2003). Urdiales et al. (2011) used collaborative control to coordinate the wheelchair navigation in an indoor environment and concluded that this kind of shared control strategy adapts to the person's needs and assists him/her when necessary. Masoud (2013) used a guidance component of the controller based on the Harmonic Potential Field approach for joint motion planning and control of dynamical mobile robots.

As mentioned in Section 1, using our proposed intelligent network to plan multi-wheelchair navigation in a crowded and dynamic environment is a NP-hard problem, and current navigation algorithms are not suitable for indoor environments that are characterized by narrow or crowded aisles. For analyzing this environment, a class of navigation networks can be considered as a weighted graph $\mathbf{G}=(\mathbf{V}, \mathbf{E}, f)$, where $\mathbf{V}$ and $\mathbf{E}$ are the sets of vertices and edges, respectively, and $f$ is a real-valued weight function $f: \mathbf{E} \rightarrow \mathbf{R}$. Vertices represent wireless nodes. An edge represents a physical routing segment between two wireless nodes and it is weighted by the time that robots need to travel. The path planning finds the shortest path from one vertex to another. For example in the weighted graph as shown in Figure 1, the shortest path between $v_{1}$ and $v_{6}$ is a path $p=\left(v_{1}, v_{2}, v_{4}, v_{6}\right)$. However, in an indoor environment, the path $p$ may be not an optimal solution, when sum of the costs involved in multiple robotic wheelchairs travel is considered. Assume that in Figure 1 there are two robots, $r_{1}$ at $v_{1}$ and $r_{2}$ at $v_{6}$ and their destinations are $v_{6}$ and $v_{1}$, respectively. If both robots move from their source to their destination simultaneously, then the optimal solutions for them will vary in the following cases.

Case 1: Every edge is bidirectional, that is, two robots can pass simultaneously a path/edge in the opposite direction. 
Case 2: Every edge is unidirectional or one-way, that is, only one robot is allowed to pass a path if two robots move in the opposite direction.

Case 3: There are obstacles, such as randomly appeared trashes and mobile objects in the way.

In case 1 , it is obvious that $\left(v_{1}, v_{2}, v_{4}, v_{6}\right)$ is the optimal path for both $r_{1}$ and $r_{2}$.

In case 2 , there are many possible situations: (1) robot $r_{1}$ with velocity vel $=2$ arrives at $v_{2}$ and robot $r_{2}$ with velocity $v e l=1.5$ arrives at $v_{4}$ at the same time. Then both robots apply to $v_{2}$ and $v_{4}$ for the access of shared resource $\left(v_{2}, v_{4}\right)$. If both nodes cannot assign the access to any of robot, then both robots will not move anymore; if both nodes let both robots be in the path without access control policies, then the robots will stop at the point where they meet; $(2)$ robot $r_{1}$ firstly arrives at $v_{2}$, then enters the path $\left(v_{2}\right.$, $v_{4}$ ) and occupy the access of the path, then robot $r_{2}$ has to wait for robot $r_{1}$ for releasing the access of the path. In this situation, robot $r_{2}$ will take 10 time units and drive itself from $v_{6}$ to $v_{1}$, and the sum of costs of both robots for both tasks is 19 time units. If node $v_{2}$ reroute robot $r_{2}$ 's path $\left(v_{1}, v_{2}, v_{4}\right.$, $\left.v_{6}\right)$ to a path $\left(v_{1}, v_{2}, v_{6}\right)$ after detecting shared resource conflict, then the sum of the costs of both robots is 18.5 time units. Obviously, the latter is a better solution for both robots.

The most complex situation is case 3, in which many factors need to be considered, such as velocity of other mobile objects and the topology of the floor plans. Therefore, using our proposed intelligent network to plan multiwheelchair navigation in a crowded and dynamic environment is similar to a situation of finding shortest paths for multiple vehicles in a dynamic network, which has been characterized as a NP-hard problem (Toth and Vigo, 2001).

According to the analysis above, when considering the limitations of narrow corridors and the dynamics of the environment, the topology of our designed network that is embedded in the indoor environments has an important influence on the performance of our prior proposed navigation method that is totally different from traditional robot navigation research based on metric map or topology map (Ji et al., 2013; Boucher et al., 2013; Faria et al., 2014; Li et al., 2013; Lankenau and Röfer, 2000; Ghrist et al., 2012; Huang and Beevers, 2005). Tian et al. (2007) have pointed out that the connectivity between nodes affect the performance of our global navigation. We believe that there is a need for flexible harmonic rules for global navigation of robotic wheelchairs, if their users booked a surgery in a hospital or there exists an emergent situation for users to pass the crowd in an indoor 
environment.

This paper extends our prior work (Tian and Feng, 2012; Jiang et al., 2011) by studying the problem of supporting multi-wheelchair harmonic navigation in an indoor environment and investigating the pros and cons of different harmonic rules in floor plans with different topologies.

\section{Overview of the navigation network}

\subsection{Goal and working principle of the navigation network}

Our prior project (Feng et al., 2008; Jiang et al., 2011; Tian and Feng, 2012) aimed to build an intelligent environment or a navigation network, which is composed of wireless visual nodes. These nodes can be installed in ceiling or higher places on the wall to support the automatic navigation of multiple robotic wheelchairs. These wheelchairs are low-cost and they require lower computation but can achieve complex tasks, such as obstacle avoidance and navigation. The architecture of our WVSN is flat as shown in Figure 2. This means that every node in this network works as a peer, and no node plays a critical role of the centralized controller.

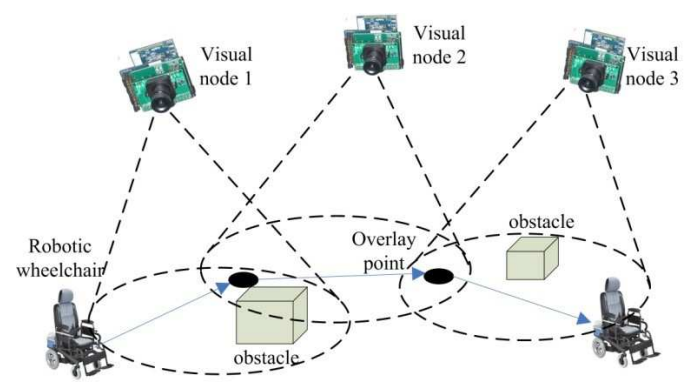

Figure 2: Navigation network

In the navigation process of a robotic wheelchair in our navigation network firstly, a user selects a destination by touching an entry in a destination option list in the interface of a Personal Digital Assistant (PDA); secondly, a message about the selected wheelchair and destination is sent to the network, and propagated to a source node that has control over the selected robotic wheelchair. This is called as commanding phase; then, a particular route between the source node to the destination node is established using a distributed navigation algorithm. This is called as searching target phase; 
after that, the wheelchair is guided in accordance with the established route to the destination node in a form of node by node movement.

\subsection{Key parts and their architectures}

The key parts and their architectures in our navigation network are as follows:

(1) Wireless visual sensor node (WVSnode), node for short. The design schema of a node is that cameras are integrated to node platform Imote2 (Nachman et al., 2008) to increase their vision. A node can run in two modes: off-line configuration and on-line computation.

- Off-line configuration. After visual nodes are calibrated, parameters for each node can be configured or set, such as labelling a node with semantic information (e.g., John's office), setting its IP address and indicating its monitoring area segments and outlet (e.g., overlay point to Alice's office).

- On-line computation. A node can achieve tasks, such as locating wheelchairs, detecting obstacles, talking to a PDA and finding paths to destinations with collaboration of other nodes in a real time manner. A robotic wheelchair can be guided node by node in an established route. Note that nodes exchange the control of a wheelchair in their overlay points.

(2) Robotic wheelchair. It is equipped with a simple and low-cost wireless sensor node. The node has low computation ability. The robotic wheelchair talks to the network and executes simple commands, such as turning left 30 degrees, going straight along a right side wall or stop.

For simplicity and low-cost, we adopt an 8-bit microcontroller Mega128 to manage sensors installed on the wheelchair to control wheelchair behaviors, and a complex programmable logic device (CPLD) is used to process the signal information from ultrasonic sensors.

For achieving goals of low-cost and simple intelligence, the behavior controller of a wheelchair is designed as a kind of reactive agent architecture (Wooldridge, 2009), as shown in Figure 3, where a wheelchair behavior is a built-in software module in an embedded control board. At the same time, a wheelchair can only execute a simple behavior that is activated according to a node command and the wheelchair perception. The real prototype of our wheelchair is shown in Figure 4. 


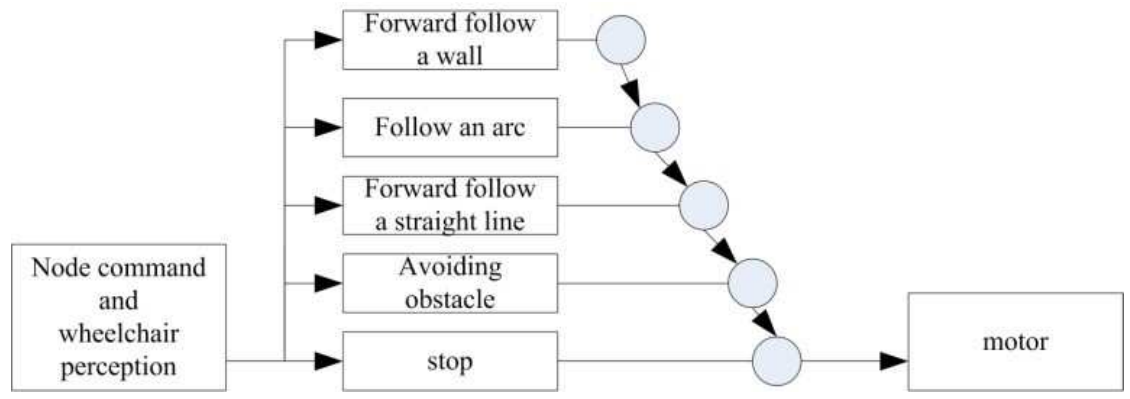

Figure 3: Reactive agent architecture for behavior control of robotic wheelchair

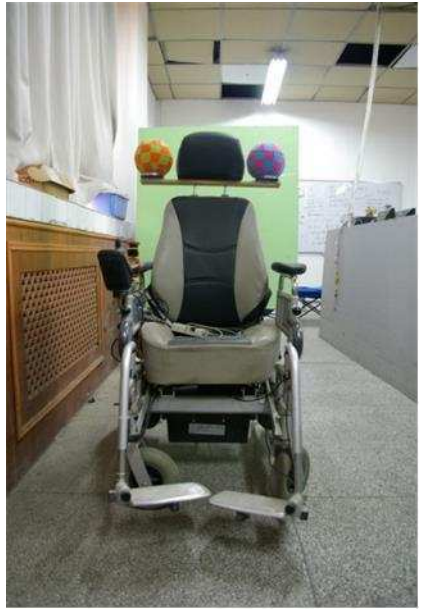

(a) Front view

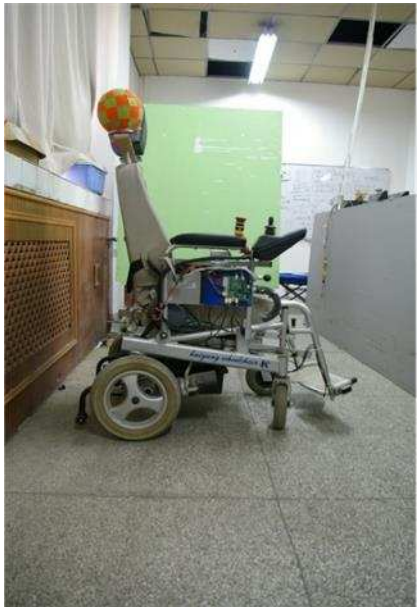

(b) Side view

Figure 4: Photos of robotic wheelchair

(3) Zigbee based wheelchair controller. It sends the commands to a robot, such as finding a specific robot and ordering a robot to move to a particular destination through the network. Its use case diagram is shown in Figure 5 ,

Other functional modules described in section 3 are implemented and their detailed information is omitted due to the size limitation of this paper. 


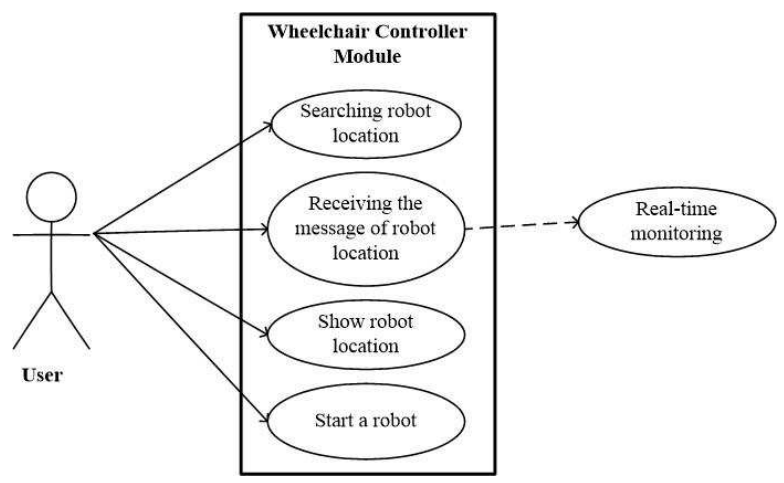

Figure 5: Use case diagram for a wheelchair controller module in a PDA

\section{Harmonic multi-wheelchair coordination in a node topological level}

In our previous work (Feng et al., 2008; Jiang et al., 2011; Tian et al., 2007), we have proposed some methods for local navigation, such as snakebased motion planning and bloom filter base navigation (bf-Navigation). We have also introduced the major phases of navigation in our prior work (Tian and Feng, 2012) and described some simulation experiments that were carried out to evaluate algorithms, such as, Random Selection Policy (RaSelP), RaSelP+Plan-Break, broad-first-search-like policy-based Plan-Forward without Punishment (BFSP+FWoutP), and broad-first-search-like policy-based Plan-Forward with Punishment (BFSP+ Plan-FWP). In this paper, we discuss other important issues that exist in low-cost wheelchair navigation: the optimization of harmonic global navigation (also called as harmonic longterm path finding), dynamic obstacle and constraints (such as imprecisely measured data and low behavior control precision). Before discussing these issues, we present some assumptions first.

Assumption I. Each wheelchair has its docking place, where it can be charged when it is in idle state.

Assumption II. The user of a wheelchair can only input his command through a PDA, and the command is broadcasted to the designated wheelchair via WVSN.

Assumption III. In an indoor environment, if a path can only be accessed by one wheelchair in one direction (shown in Figure 6(a)) at a time, then a 
node adopts the policy subscribed according to the priority of the wheelchair. If a path can be accessed by multiple wheelchairs in bi-direction (shown in Figure 6(b)) at a time, then the node coordinates multi-wheelchairs to pass synchronously in complying with the rules of the right hand.

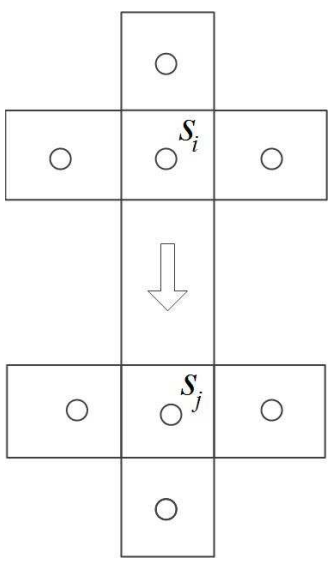

(a) Single direction

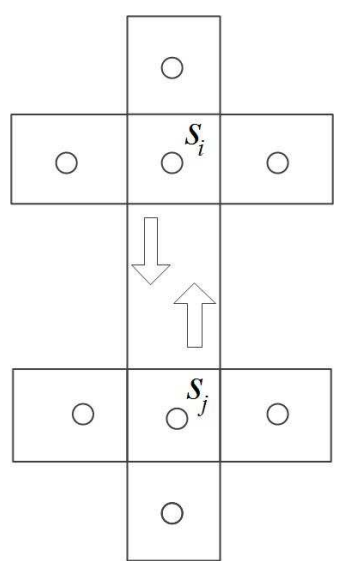

(b) Bi-direction

Figure 6: Cases of single direction and bi-direction

Note that false positive probability of using bf-Navigation is proven (Yuanxiang et al., 2009; Jiang et al., 2011). So, bf-Navigation can ensure that every node knows the potential path to the target node that the wheelchair or a user queried, after asking for help from nodes with two hops. Therefore, from the topological view, bf-Navigation is adopted in order to reduce the set of searched nodes.

\subsection{Navigation algorithm and harmonic rules}

Inspired by the idea of replacing physical paths with communication routing (Tian et al., 2007), the solution to global navigation in an indoor environment is transformed into the problem of designing a harmonic navigation protocol that includes operations of query, confirmation, and execution. In the navigation protocol, we assign each wheelchair a priority, and ensure that a wheelchair with higher priority passes first. Three harmonic rules are as follows:

A. Highest priorities to humans (navigation algorithm with highest priority to humans, NAHPH) 
Rule A1) If there is a moving object or other wheelchairs with higher priority on a wheelchair's way to its destination, the wheelchair stops and waits until the moving object passes.

B. Highest priorities to wheelchairs (navigation algorithm with highest priority to wheelchairs, NAHPW)

Rule B1) If two wheelchairs are involved in a conflict, the navigating node in the navigation network randomly chooses one of them to assign it the right to pass the path.

C. Flexible priorities to wheelchairs (navigation algorithm with the flexible harmonic rules)

Rule C1) There are three-level of priorities, top-level, mid-level, and lower level. The wheelchair with top-level priority can pass and alarm the crowd with its light, while humans always hold mid-level priority and wheelchairs with routine tasks have lower level priority.

In addition to above harmonic rules, a rule of half-half chance for wheelchairs is also adopted. This is, if two wheelchairs with the same level of priority are involved in a conflict, the navigating node in the navigation network randomly chooses one of them to occupy the right to access the path. We can describe the running mode of our approach in term of four phases. These phases are:

1) The commanding phase: After a user inputs his command by a PDA equipped with a GainZ (Tian and Feng, 2012), the command is sent to a wheelchair via a node that is the nearest to the wheelchair. This phase is called as the commanding phase.

2) The searching target phase: After receiving the order, the wheelchair sends a query to search the target node through wireless communication between nodes. In this phase, there are three important issues that should be considered.

(a) The strateqy of the transmitting search message: Based on our prior work (Jiang et al., 2011; Tian et al., 2007), the Bloom Filter-based navigation algorithm is adopted in the second phase for searching potential paths to a target node.

(b) A conflict prediction technique is adopted. In which, considering the moving objects in real-world applications/environments, the influence or interference between moving objects (wheelchairs or obsta- 
cles) should be considered not only during the second, but also during the third and fourth phases. In the second phase, a technique is used to predict potential conflicts between wheelchairs or between wheelchairs and dynamic obstacles, in which every node computes the time that an object arrives in its region and the duration they occupy, with an assumption that the speed of all moving objects in the environment is constant. To estimate the cost that a wheelchair passes a path, we adopt Plan-Forward-With-Punish-Priority (Plan-FWPP). Plan-FWPP means a node uses a penalty policy to compute its weight that combines the pure-execution-time (PET). The wheelchair with the latest/earliest time among the wheelchairs involved in a same conflict can have right to access the path, while considering their priorities. This means that the latest time of each wheelchair is calculated according to

(i) $\min _{l=1}^{L_{i}}\left(T^{r}(s, l)+T^{r}(l, i)+\max _{r 1}^{R^{c}} T^{r 1}(i, l)\right)$, if their priorities are same;

(ii) $\min _{l=1}^{L_{i}}\left(T^{r}(s, l)+T^{r}(l, i)+T_{\min }^{r 1}(i)\right)$, if the priority of a wheelchair $r 1$ is higher than other wheelchairs involved in the same conflict and $\left|\min _{r \neq r 1}^{R^{c}} T^{r}(i)-T_{\min }^{r 1}(i)\right|<\frac{\underset{l}{L_{i}^{r 1}} T^{r 1}(l, i)}{3}$.

Therefore, when a query message is transmitted or broadcasted, the weights of each path are handed over and computed one by one as follows:

$T_{\min }^{r}(s, i)=\left\{\begin{array}{cc}\min _{l=1}^{L_{i}}\left(T^{r}(s, l)+T^{r}(l, i)\right), & \text { if there is no conflicts } \\ \operatorname{Lin}_{i=1}\left(T^{r}(s, l)+T^{r}(l, i)+\underset{r 1}{R^{c}} T^{r 1}(i, l)\right), & \text { if meet conflict conditions } \\ \min _{l=1}^{L_{i}}\left(T^{r}(s, l)+T^{r}(l, i)+T_{\min }^{r 1}(i)\right), & \text { if there exist } r 1 \succ \text { others and } \\ \min _{\substack{r 1 \\ \text { in }}}^{r 1}(l, i) \\ 3\end{array}\right.$

where $l=1, \ldots, L_{i}^{r} ; i=1, \ldots, N ; r=1, \ldots, R ; N$ is the total number of nodes in the WVSN; $i$ represents the $i$-th node; $L_{i}^{r}$ is the number of one hop neighbors around the $i$-th node; $R$ represents the total number of wheelchairs in the WVSN; $r$ represents the $r$-th robot/wheerlchair; $s$ denotes the source node. $T^{r}(s, i)$ indicates the 
time cost that wheelchair $r$ travels from node $s$ to node $i$. Obviously, $T_{\min }^{r}(s, i)$ means that to find the shortest route from $s$ to $i$ is to find the nearest neighbors of the $i$-th node and the shortest path from node $s$ to the set of neighbor nodes of node $i$. While the weight, $T^{r}(s, e)$, of every path to the target $e$ for wheelchair $r$ is computed, and hops of every path are counted during the searching phase. $\succ$ denotes the operator of priority. For instance, $r 1 \succ r 2$ means that the priority of $r 1$ is higher than that of $r 2$. If some wheelchairs have same priority, then one with the latest time to arrive the node wins.

The conflict measuring conditions are:

(i) the moving direction of $r$ and $r 1$ is opposite during passing the path between node $i$ and node $l$;

(ii) the path from the node to node $i$ is single path;

(iii) there exists $r 1 \in R^{c}$ and

$$
T_{\min }^{r 1}\left(s^{r}, l\right)-T_{\min }^{r 1}\left(s^{r 1}, l\right)>T^{r 1}(i, l)
$$

(iv) there exists $r 1 \succ r \exists R^{c}$ and

$$
\left|\min _{r \neq r 1}^{R^{c}} T^{r}(i)-T_{\min }^{r 1}(i)\right|<\frac{\min _{l}^{L^{r 1}} T^{r 1}(l, i)}{3}
$$

where $r \neq r 1,0<r 1 \in R^{c}, R^{c}$ is a set of wheelchairs that will pass the path from node $i$ to node $l$ and satisfies the conflict conditions in Equation (11); $s^{r}$ represents the source node for wheelchair $r$. $T^{r}(i)$ represents the time that wheelchair $r$ arrive at node $i$. $T^{r}$ min $(i)$ represents the shortest time that wheelchair $r$ arrive at node $i$. Equation (2) introduces the conflict measure conditions, called as conflict conditions. If there are no conflicts, $T^{r}{ }_{\min }(s, i)$ is equal to $\min _{l=1}^{L_{i}}\left(T^{r}(s, l)+T^{r}(l, i)\right)$; otherwise, a penalty item $\max _{r 1}^{R^{c}} T^{r 1}(i, l)$ or $T^{r 1}{ }_{\text {min }}^{l=1}(i, l)$ is added. This strategy results in a wheelchair considering the cost of potential conflicts during search of the optimal path.

(c) The stop conditions of searching are: (i) Hop is greater than N, and (ii) the count of timer in the source node is greater than the threshold, $T h_{D}$, that is usually set as two minutes. 
3) Route confirmation phase: During this phase, the target node feeds back the confirmation message to the wheelchair through the sensor network in order to select an optimal route for wheelchair $r$. A broad-first-searchlike policy (BFSP) (Tian and Feng, 2012) is adopted in this paper. This policy is like a kind of dynamic-programming solution provided by backforward induction. Every node in the shortest path from the target node to the source node determines the shortest predecessor node, $i_{\text {pred }}$, within one hop, considering the weight and hop information that are aggregated during second phase. After this process, each node in the shortest path commits to help the wheelchair to finish its navigation task.

4) Navigation phase. The wheelchair that is approaching the target node is detected, recognized and tracked by each WVSnode that had committed to the routing task during the third phase, while the wheelchair sends and receives messages through its equipped node. In addition, the real-world environment is full of the multiple moving objects and static objects that can suddenly appear in a wheelchair's way. For example, trash cans can be placed suddenly in a wheelchair's way. So, a common rule for robotic wheelchairs is formulated.

CommonRule: If there is a static object in a wheelchair's way to its destination and the node detects that the path is feasible, the wheelchair will find solutions to pass the obstacle. If it fails to do so, it will return to the nearest node and choose another potential path to the target node; Note that the commonRule is applied to three kinds of harmonic rules.

\subsection{Experiments and results}

For investigating the harmonic rules, we consider three kind of scenarios. In the first scenario, wheelchairs have higher priority than humans. In the second, humans have higher priority than the wheelchairs. In the third, priority of a wheelchair is changeable according to the emergent degree of its task.

For comparing the advantages and disadvantages of the proposed navigation with different harmonic rules, we have developed a simulation platform as shown in Figure 7. The simulation platform mimics nodes' and wheelchairs' behaviors in various floor plans. In Figure 7, each colored rectangle represents a moving wheelchair or a moving obstacle, whereas each blue circle denotes a wireless visual sensor node, while bricks or walls are represented by brown or grey connected rectangles. The area of each floor 
plan is $30 \mathrm{~m}^{*} 30 \mathrm{~m}$. Note that, Figure 7 (a) shows a floor plan of two buildings connected by a corridor and there is no circle in its topology. Figure 7(b) shows a floor plan of two buildings connected by a corridor and there are three tie-like circles in its topology. Figure 7(c) shows a floor plan with four crosses in its topology. Figure 7(d) shows a floor plan with a two dimension grid. Figure $7(\mathrm{e})$ shows a floor plan with a rectangle topology. Figure $7(\mathrm{f})$ shows a floor plan with a T-shape topology. Figure $7(\mathrm{~g})$ shows a floor plan with nine cubes, and Figure 7(h) shows a floor plan with a I-shape topology. Figures 7(a)-(h) are represented by 2bconR, 3tie, 4cross, 2d-grid, Rectangle, T, 9cube and I-shape, respectively.

The Plan-FWPP with highest priority for humans (Plan-FWPPHPH) and the Plan-FWPP with flexible harmonic rule are carried out in the topology of each floor plan twelve times, and the number of moving obstacles is set as $3,7,12,17$ and 22 , respectively. The results of the average delay of execution time that all wheelchairs finished their tasks are shown in Table 1 and Table 2, respectively. Note that the tasks are randomly generated and assigned to a wheelchair by the simulator, once the wheelchair finished its current task.

It is obvious that the average delay of the execution time of every wheelchair is small, after applying the navigation algorithm with highest priority for wheelchairs. So, the Plan-FWPP with highest priority for wheelchairs (Plan$F W P P H P W$ ) are carried out on some floor plans on which Plan-FWPPHPH did not perform well. The results are shown in Table 3 ,

Table 1: Average delay of each task for three wheelchairs after employing the flexible harmonic rules

\begin{tabular}{lccccc}
\hline Topology of & \multicolumn{5}{c}{ Num. of Person } \\
\cline { 2 - 6 } \multicolumn{1}{c}{ floor plan } & 2 & 7 & 12 & 17 & 22 \\
\hline Rectangle & 1.246064 & 3.289060 & 5.065047 & 7.774448 & 24.222298 \\
I-shape & 2.150243 & 6.366557 & 12.542470 & 25.821930 & 50.063330 \\
T & 2.428571 & 8.712842 & 29.179350 & 68.779900 & 74.190140 \\
3tie & 1.408033 & 4.735668 & 9.834129 & 23.589740 & 70.953490 \\
2bconR & 1.744048 & 6.375369 & 14.666960 & 38.802310 & 110.165600 \\
4cross & 1.115702 & 3.370649 & 6.855518 & 13.203970 & 22.601210 \\
9cube & 0.593279 & 2.028237 & 3.768678 & 6.502592 & 10.200620 \\
2d-grid & 0.380811 & 1.622449 & 1.609758 & 2.518980 & 3.397064 \\
\hline
\end{tabular}

Table 1 and Table 2 indicate that, with the increase in number of moving 
persons in the various topologies of floor plan, the average delay time of a task carried by the wheelchair increases. At the same time, we observe that the average delay time of tasks carried out by the wheelchairs employing the flexible harmonic rules are smaller than the one where wheelchairs are employing Plan-FWPPHPH. However, Table 3 shows that, for the worse situations, the average delays that wheelchairs take to finish all tasks by employing Plan-FWPPHPW is relatively small for different topologies of floor plans. It should be mentioned that, due to flexible harmonic rules, the wheelchair with the top-level priority in the simulation has no task execution delay. This means that the wheelchair user can deal with the emergency situation or meet the doctor and attend the appointment on time.

Table 2: Average delay of each task for three wheelchairs after giving the highest priority to humans

\begin{tabular}{lccccc}
\hline Topology of & \multicolumn{5}{c}{ Num. of Persons } \\
\cline { 2 - 6 } \multicolumn{1}{c}{ floor plan } & 2 & 7 & 12 & 17 & 22 \\
\hline Rectangle & 0.963634 & 2.21855 & 3.432622 & 5.103389 & 12.79727 \\
I-shape & 1.57485 & 4.097119 & 7.742161 & 12.9849 & 20.44698 \\
$\mathrm{~T}$ & 2.198093 & 6.622137 & 17.48124 & 28.16011 & 20.58777 \\
3tie & 1.06915 & 3.124986 & 6.560556 & 13.26531 & 24.7315 \\
2bconR & 1.266044 & 4.233871 & 8.627232 & 17.2238 & 32.1627 \\
4cross & 0.835041 & 2.194093 & 4.415239 & 7.574163 & 11.47761 \\
9cube & 0.469956 & 1.373674 & 2.6528 & 4.037454 & 6.202736 \\
2d-grid & 0.311891 & 1.096579 & 1.139165 & 1.621414 & 2.239351 \\
\hline
\end{tabular}

Table 3: Average delay of each task for three wheelchairs after giving the highest priority to them

\begin{tabular}{cc}
\hline Topology of floor plan & Num. of Person $(=22)$ \\
\hline I-shape & 0.465658 \\
T & 0.253731 \\
3tie & 0.355839 \\
2bconR & 0.459184 \\
\hline
\end{tabular}

From these experimental results, we can conclude that the features of the topology of the floor plans have an important influence on the service time of each wheelchair, especially in some extreme situations, such as $\mathrm{T}$ or $2 \mathrm{bconR}$. 
Suggestions for designers and researchers of the robotic wheelchair are that they should create balance between the human safety and rights of wheelchair users to access the critical path.

Suggestions for wheelchair users are 1) harmonic rules depend on collaborative interaction between humans and wheelchair users; 2) being an early bird can catch the deadline; 3) be familiar with the environment, especially the topology of floor plan, that will be visited. Suggestions for the building owners and architects are that they should leave spaces or design a specialized route/area for wheelchair users for the upcoming ageing society.

\section{Real-world experiments}

Real-world experiments for testing harmonic navigation were conducted. First, we test the performance of wireless visual sensor node. As shown in Table 4, the experimental results revealed that the average of inquiring frame rate of a wireless visual node is 22 frames per second (fps). The rate of recognizing one and two wheelchairs are up to ten and $4 \mathrm{fps}$, respectively. The average velocity of the wheelchair is $0.330 \mathrm{~m} / \mathrm{s}$, while the minimum velocity is $0.324 \mathrm{~m} / \mathrm{s}$. The average enquiry time for a query is $0.068827 \mathrm{~s}$.

In second experiment, a performance test is carried out ten times. In which, a wheelchair goes straight 2 meters starting from node 05 , turns right 90 degree, and then goes straight 6 meters from node 02 to node 00 . The floor plan of the experimental environment is shown in Figure 8, where red points indicate the location where nodes are installed and blue links mean that there is a unidirectional path between nodes, and a pen installed on the wheelchair traces each robot's tracks. The performance of the improved wheelchair is shown in Table 5. It can be observed from Table 5 that the maximal distance between the expected end point and the actual end point is $13.5 \mathrm{~cm}$, while the minimal distance from the expected end point is $4.5 \mathrm{~cm}$. The maximal distance between the two farthest tracks when a wheelchair turns 90 degree is $16 \mathrm{~cm}$. The maximal distance between the two farthest tracks when a wheelchair goes a straight line is $16 \mathrm{~cm}$.

In the third experiment, we take the velocity experiment of a wheelchair as a simple comparison between the real results and theoretical results. An experiment on a real physical environment is carried out. In which, the wheelchair goes a $3 \mathrm{~m}$ straight line five times when setting the wheelchair velocity as $0.330 \mathrm{~m} / \mathrm{s}$, and we recorded the time taken by each test. The results are shown in Table 6, In Table 6, the average velocity of the wheelchair 
Table 4: Performance index of the improved wheelchair

\begin{tabular}{ll}
\hline Items & Average time $(\mathrm{s})$ \\
\hline Detecting a wheelchair & 0.095818 \\
Detecting the shape of an obstacle & 0.096714 \\
Detecting two wheelchairs simultaneously & 0.130531 \\
detecting whether there exists an obstacle & 0.0654689 \\
\hline
\end{tabular}

Table 5: Positioning errors at the end point of each track

\begin{tabular}{|c|c|c|c|c|c|c|c|c|c|c|c|}
\hline$i$-th test $^{1}$ & 1 & 2 & 3 & 4 & 5 & 6 & 7 & 8 & 9 & 10 & averag \\
\hline $\begin{array}{l}\text { Positioning } \\
\text { error }(\mathrm{cm})^{2}\end{array}$ & 4.5 & 8.1 & 9.1 & 7.2 & 8.1 & 13.5 & 7.0 & 11.0 & 8.0 & 10.3 & 8.68 \\
\hline
\end{tabular}

is $0.329 \mathrm{~m} / \mathrm{s}$. We observed that the velocity values of each test is slightly different from each other. On the contrary, in our simulation environment, the velocity of a wheelchair is set as a constant value, such as $0.330 \mathrm{~m} / \mathrm{s}$, in the initialization phase of each simulation, because we did not consider detailed information, such as the delay of robotic wheelchair executor, the ground friction, and sensor measurement error. So, there is definitely no variation of velocity in each test. The simulation results should be the same value as what we expected if there is no random obstacles in the wheelchair's way.

The fourth experiment is shown in Figure 9, in which an automatic navigation process of a robotic wheelchair is presented. The wheelchair starts to move to the destination that is given by the user's commands via the PDA. The commands are sent that one wheelchair in node 10 goes to node 00 (the floor plan of this experiment is shown in Figure 8). The experiment was carried out twice. In the first test, the wheelchair cannot pass the path from node 05 to node 03 , which is occupied by two persons seated on chairs. Then, the wheelchair chooses another feasible path from node 12 to node 00. But after facing a person (seen in Figure $9(\mathrm{a}(1) \mathrm{a}(4))$, the wheelchair still slowly approaches its destination by following the harmonic rule of dealing with the

Table 6: Results of velocity experiment

\begin{tabular}{lllllll}
\hline & 1 & 2 & 3 & 4 & 5 & average \\
\hline time taken(s) & 9.11 & 9.38 & 8.98 & 8.91 & 9.23 & 9.122 \\
Speed(m/s) & 0.329 & 0.320 & 0.334 & 0.337 & 0.325 & 0.329 \\
\hline
\end{tabular}


emergent task, while the person slowly moves backward. In the second test, the wheelchair moves with the ability to avoid obstacles (a trash bin) (seen in Figure 9(b)).

The fifth experiment on path finding involving a conflict between two wheelchairs, one in node 10 and another in node 00, is carried out. The results show that the wheelchairs automatically navigate via our wireless visual sensor network. Figure 10 shows a series of video frames from this experiment.

Based on our implementation and the experiment results, the practical implications for robotic wheelchair researchers and engineers are (1) issues about safety should be considered during the design and implementation phases. For example, it is necessary to install an emergency stop button on the wheelchair, especially during testing phase; (2) the selection of sensor types (e.g., ultrasonic sensors and infrared sensors) and their precision is crucial for control algorithms and strategies, especially when low-cost is taken as a primary goal; (3) how to assign tasks between a network and a robotic wheelchair. The reactive agent architecture for behavior control of a robotic wheelchair managed to control the low-level behaviors of a robotic wheelchair, while the task of global navigation was carried out by a wireless sensor network. This kind of 'divide-and-conquer' strategy works well in our implementation; (4) the detection speed and precision of a visual node is very important. There is still space for improvement for it; (5) the off-line configuration system for wireless sensor should be better integrated with the simulation functions of navigation protocol. This helps us to detect many problems during protocol design.

\section{Conclusions}

In this paper, we focus on the problem of supporting harmonic navigation of low-cost multi-wheelchair in an indoor environment that has characteristics of narrow or crowded aisles, with low-cost constraint features, such as imprecisely measured data and low precision of behavior control. Through extensive quantitative simulations, we explore various floor plan topologies and different values for the system parameters, and the simulation results demonstrate that they have important influence on the performance of global navigation method and harmonic rules. The proposed navigation method with flexible harmonic rules can help establish trade-off for the access of the critical paths between humans and robotic wheelchairs. At the same time, 
the pros and cons of three different harmonic rules are discussed. This will benefit not only the indoor applications, such as smart home and health care center, but also the designers or researchers of robotic wheelchairs, building owners and architects. The proposed approach is capable of converting lowcost wheelchairs into intelligent wheelchairs without requiring fundamental design changes.

There are still outstanding issues to be addressed in this domain, for example how to devise an optimal approach to estimate the weight of each path in a dynamic environment, especially when the speed of a wheelchair is not constant. In addition, it is a challenging task for us to prove how a WVSN can be simplified to a network with a characteristic of low edge connectivity to make bf-Navigation based routing algorithms more efficient, and incorporate it into the information management of service-oriented applications (Huergo et al., 2014; Lane et al., 2014) in intelligent buildings.

\section{ACKNOWLEDGMENT}

This work was supported in part by the National Key Tech. R\&D Program of China under Grant No. 2013BAK09B01, the funding of the costshared project (60711130071) supported by International Joint Project of the Royal Society and National Science Foundation of China (NSFC), $863 \mathrm{Hi}$ tech program (2006AA04Z222), the National Science Foundation of China under Grant No. 61472315, Microsoft-XJTU Joint Lab and China Scholarship Council under Grant No. [2013]3018. This research is also carried out as a part of GREENet project which is supported by a Marie Curie International Research Staff Exchange Scheme Fellowship within the 7th European Community Framework Programme under grant agreement No 269122. Thanks for other teammates, Liang Song, Die Hu, Xiao Tian, Huo Jiang, Yaobin Liang, Jiaquan Su, Kaibin, Huang, Jintao Xu, Daqing yi, Gang Cheng, etc.

\section{References}

Akyildiz, I. F., Melodia, T., Chowdhury, K. R., 2007. A survey on wireless multimedia sensor networks. Computer networks 51 (4), 921-960.

Baeg, S.-H., Park, J.-H., Koh, J., Park, K.-W., Baeg, M.-H., 2007. Building a smart home environment for service robots based on rfid and sensor networks. In: Control, Automation and Systems, 2007. ICCAS'07. International Conference on. IEEE, pp. 1078-1082. 
Batalin, M., Sukhatme, G. S., Hattig, M., et al., 2004. Mobile robot navigation using a sensor network. In: Robotics and Automation, 2004. Proceedings. ICRA'04. 2004 IEEE International Conference on. Vol. 1. IEEE, pp. 636-641.

Bhattacharya, S., Atay, N., Alankus, G., Lu, C., Bayazit, O. B., Roman, G.-C., 2006. Roadmap query for sensor network assisted navigation in dynamic environments. In: Distributed Computing in Sensor Systems. Springer, pp. 17-36.

Boucher, P., Atrash, A., Kelouwani, S., Honoré, W., Nguyen, H., Villemure, J., Routhier, F., Cohen, P., Demers, L., Forget, R., et al., 2013. Design and validation of an intelligent wheelchair towards a clinically-functional outcome. J. Neuroeng. Rehabil 10 (1), 58.

Cao, T., Wu, X., Guo, J., Yu, S., Xu, Y., 2009. Abnormal crowd motion analysis. In: Robotics and Biomimetics (ROBIO), 2009 IEEE International Conference on. IEEE, pp. 1709-1714.

Chen, X., Xu, Y., Li, Q., 2013. Application of adaptive extended kalman smoothing on ins/wsn integration system for mobile robot indoors. Mathematical Problems in Engineering 2013.

Del Castillo, G., Skaar, S., Cardenas, A., Fehr, L., 2006. A sonar approach to obstacle detection for a vision-based autonomous wheelchair. Robotics and Autonomous Systems 54, 967-981.

Faria, B. M., Reis, L. P., Lau, N., 2014. A survey on intelligent wheelchair prototypes and simulators. In: New Perspectives in Information Systems and Technologies, Volume 1. Springer, pp. 545-557.

Feng, Z., Lu, N., Jiang, P., 2008. Posterior probability measure for image matching. Pattern Recognition 41 (7), 2422-2433.

Ghrist, R., Lipsky, D., Derenick, J., Speranzon, A., 2012. Topological landmark-based navigation and mapping. University of Pennsylvania, Department of Mathematics, Tech. Rep 8.

Guzzi, J., Giusti, A., Gambardella, L. M., Theraulaz, G., Caro, G. A. D., 2013. Human-friendly robot navigation in dynamic environments. In: Proceedings - IEEE International Conference on Robotics and Automation. pp. $423-430$. 
Hamagami, T., Hirata, H., 2004. Development of intelligent wheelchair acquiring autonomous, cooperative, and collaborative behavior. In: Systems, Man and Cybernetics, 2004 IEEE International Conference on. Vol. 4. IEEE, pp. 3525-3530.

Huang, W. H., Beevers, K. R., 2005. Topological mapping with sensinglimited robots. In: Algorithmic Foundations of Robotics VI. Springer, pp. $235-250$.

Huergo, R. S., Pires, P. F., Delicato, F. C., Costa, B., Cavalcante, E., Batista, T., 2014. A systematic survey of service identification methods. Service Oriented Computing and Applications 8 (3), 199-219.

Ji, Y., Hwang, J., Kim, E. Y., 2013. An intelligent wheelchair using situation awareness and obstacle detection. Procedia-Social and Behavioral Sciences 97, 620-628.

Jiang, P., Feng, Z., Cheng, Y., Ji, Y., Zhu, J., Wang, X., Tian, F., Baruch, J., $\mathrm{Hu}$, F., 2011. A mosaic of eyes. Robotics \& Automation Magazine, IEEE 18 (3), 104-113.

Kobayashi, Y., Suzuki, R., Sato, Y., Arai, M., Kuno, Y., Yamazaki, A., Yamazaki, K., 2013. Robotic wheelchair easy to move and communicate with companions. In: CHI'13 Extended Abstracts on Human Factors in Computing Systems. ACM, pp. 3079-3082.

Kuderer, M., Kretzschmar, H., Burgard, W., 2013. Teaching mobile robots to cooperatively navigate in populated environments. In: Intelligent Robots and Systems (IROS), 2013 IEEE/RSJ International Conference on. IEEE, pp. 3138-3143.

Kuno, Y., Yoshimura, T., Mitani, M., Nakamura, A., 2003. Robotic wheelchair looking at all people with multiple sensors. In: Multisensor Fusion and Integration for Intelligent Systems, MFI2003. Proceedings of IEEE International Conference on. IEEE, pp. 341-346.

Lam, C.-P., Chou, C.-T., Chiang, K.-H., Fu, L.-C., 2011. Human-centered robot navigationtowards a harmoniously human-robot coexisting environment. Robotics, IEEE Transactions on 27 (1), 99-112. 
Lane, S., Gu, Q., Lago, P., Richardson, I., 2014. Towards a framework for the development of adaptable service-based applications. Service Oriented Computing and Applications 8 (3), 239-257.

Lankenau, A., Röfer, T., 2000. Smart wheelchairs - state of the art in an emerging market. KI 14 (4), 37-39.

Li, Q., Rus, D., 2005. Navigation protocols in sensor networks. ACM Transactions on Sensor Networks (TOSN) 1 (1), 3-35.

Li, R., Oskoei, M. A., McDonald-Maier, K. D., Hu, H., 2013. Ros based multi-sensor navigation of intelligent wheelchair. In: Emerging Security Technologies (EST), 2013 Fourth International Conference on. IEEE, pp. 83-88.

Li, W., Shen, W., 2011. Swarm behavior control of mobile multi-robots with wireless sensor networks. Journal of Network and Computer Applications 34 (4), 1398-1407.

Louloudi, A., Mosallam, A., Marturi, N., Janse, P., Hernandez, V., 2010. Integration of the humanoid robot nao inside a smart home: A case study. In: Proceedings of the Swedish AI Society Workshop (SAIS). Linköping Electronic Conference Proceedings. Vol. 48. pp. 35-44.

Lu, T., Yuan, K., Zou, W., Hu, H., 2006. Smooth path planning for intelligent wheelchair based on human-machine interaction. In: Information Acquisition, 2006 IEEE International Conference on. IEEE, pp. 988-993.

Masoud, A., 2013. A harmonic potential field approach for joint planning and control of a rigid, separable nonholonomic, mobile robot. Robotics and Autonomous Systems 61, 593-615.

Matsumoto, O., Komoriya, K., Hatase, T., Nishimura, H., 2006. Autonomous traveling control of the" tao aicle" intelligent wheelchair. In: Intelligent Robots and Systems, 2006 IEEE/RSJ International Conference on. IEEE, pp. 4322-4327.

Mihailidis, A., Elinas, P., Boger, J., Hoey, J., 2007. An intelligent powered wheelchair to enable mobility of cognitively impaired older adults: An anticollision system. Neural Systems and Rehabilitation Engineering, IEEE Transactions on 15 (1), 136-143. 
Murakami, Y., Kuno, Y., Shimada, N., Shirai, Y., 2001. Collision avoidance by observing pedestrians' faces for intelligent wheelchairs. In: Intelligent Robots and Systems, 2001. Proceedings. 2001 IEEE/RSJ International Conference on. Vol. 4. IEEE, pp. 2018-2023.

Nachman, L., Huang, J., Shahabdeen, J., Adler, R., Kling, R., 2008. Imote2: Serious computation at the edge. In: Wireless Communications and Mobile Computing, 2008. IWCMC'08. International Conference on. IEEE, pp. 1118-1123.

O'Hara, K. J., Walker, D. B., Balch, T. R., 2008. Physical path planning using a pervasive embedded network. Robotics, IEEE Transactions on 24 (3), $741-746$.

Okorn, B., Xiong, X., Akinci, B., Huber, D., 2010. Toward automated modeling of floor plans. In: Proceedings of the Symposium on 3D Data Processing, Visualization and Transmission. Vol. 2.

Payton, D. W., Daily, M. J., Hoff, B., Howard, M. D., Lee, C. L., 2001. Pheromone robotics. In: Intelligent Systems and Smart Manufacturing. International Society for Optics and Photonics, pp. 67-75.

Simpson, R., 2005. Smart wheelchairs: A literature review. Journal of rehabilitation research and development 42 .

Sisbot, E. A., Marin-Urias, L. F., Alami, R., Simeon, T., 2007. A human aware mobile robot motion planner. Robotics, IEEE Transactions on $23(5), 874-883$.

Tao, Y., Wang, T., Wei, H., Chen, D., 2009. A behavior control method based on hierarchical pomdp for intelligent wheelchair. In: Advanced Intelligent Mechatronics, 2009. AIM 2009. IEEE/ASME International Conference on. IEEE, pp. 893-898.

Tian, F., Deng, Q., Feng, Z., Jiang, P., 2007. Artificial immune network based robot behavior coordination under wireless network. In: Pervasive Computing and Applications, 2007. ICPCA 2007. 2nd International Conference on. IEEE, pp. $72-77$.

Tian, F., Feng, Z.-R., 2012. Coordinating navigation of multi-wheelchairs in indoor environments with wireless visual sensor network. In: Distributed 
Smart Cameras (ICDSC), 2012 Sixth International Conference on. IEEE, pp. 1-6.

Tomari, R., Kobayashi, Y., Kuno, Y., 2014. Socially acceptable smart wheelchair navigation from head orientation observation. International journal on smart sensing and intelligent systems 7 (2), 630-643.

Toth, P., Vigo, D., 2001. The vehicle routing problem. Society for Industrial and Applied Mathematics.

Trieu, H., Nguyen, H. T., Willey, K., 2008. Shared control strategies for obstacle avoidance tasks in an intelligent wheelchair. In: Engineering in Medicine and Biology Society, 2008. EMBS 2008. 30th Annual International Conference of the IEEE. IEEE, pp. 4254-4257.

Urdiales, C., Fernández-Carmona, M., M. Peulaa, J., Cortés, U., Annichiaricco, R., Caltagirone, C., Sandoval, F., 2011. Wheelchair collaborative control for disabled users navigating indoors. Artificial Intelligence in Medicine 52, 177-191.

Wooldridge, M., 2009. An introduction to multiagent systems. John Wiley $\&$ Sons.

Wu, H., Qu, S., Xu, D., Chen, C., 2014. Precise localization and formation control of swarm robots via wireless sensor networks. Mathematical Problems in Engineering 2014.

Yuan, K., 2009. Current situation and trend of intelligent wheel chair research. China Medical Device Information 15 (1).

Yuanxiang, J., Ping, j., Jin, Z., Xiaonian, W., 2009. Design of multiple bloomfilters based on error expectation. In: Wireless Mobile and Computing, Proceeding of IET International Conference on. IEEE, pp. 753-756. 


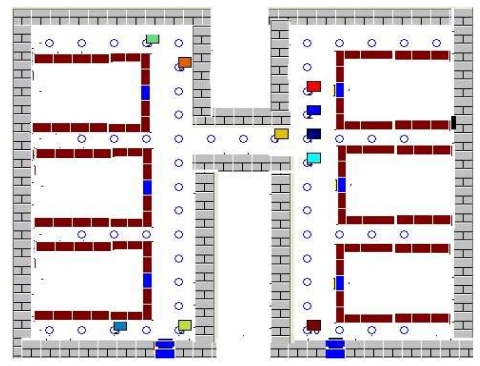

(a) 2-building connection floor plan

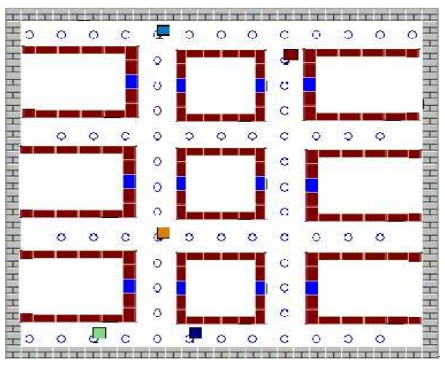

(c) four cross

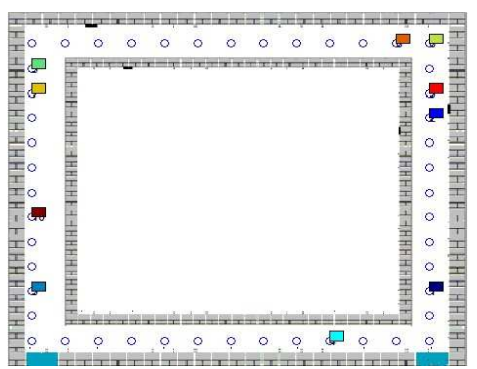

(e) Rectangle floor plan

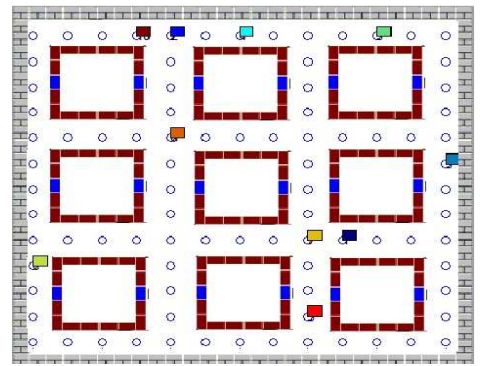

(g) nine cubes floor plan

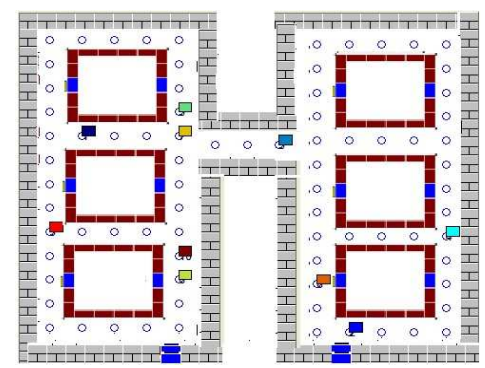

(b) 3-tie shape floor plan

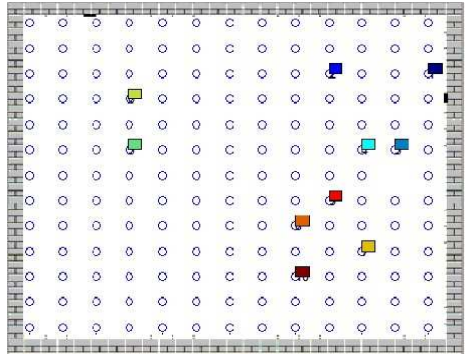

(d) 2-dimension grid floor plan

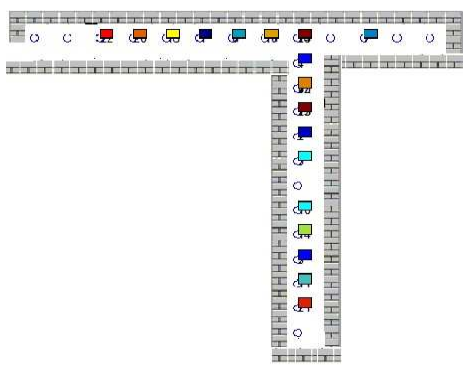

(f) T-shape floor plan

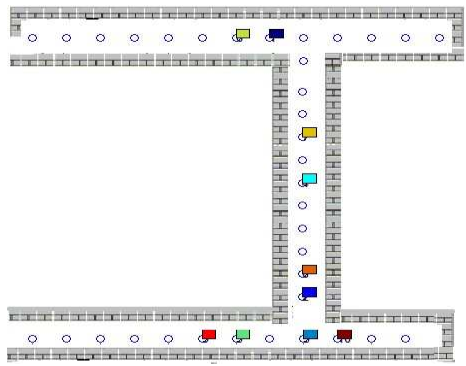

29

Figure 7: Snapshots of simulation floor plans with different topologies 


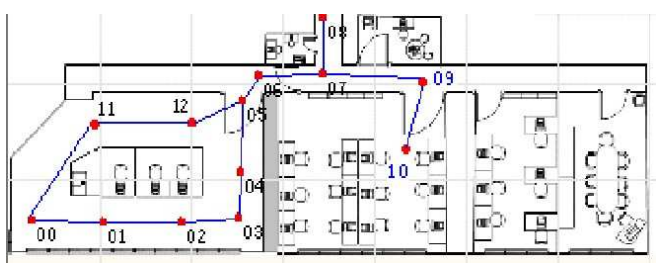

Figure 8: A part of floor plan which build the navigation network

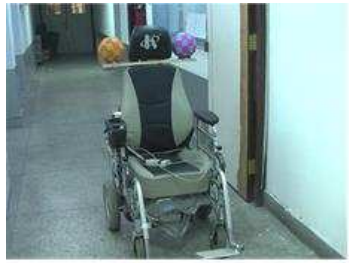

(1)

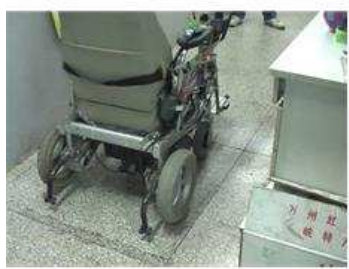

(4)

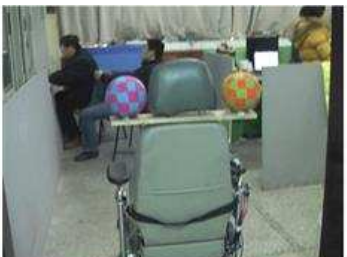

(2)

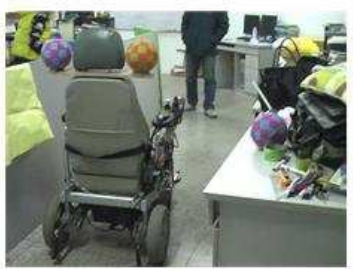

(5)

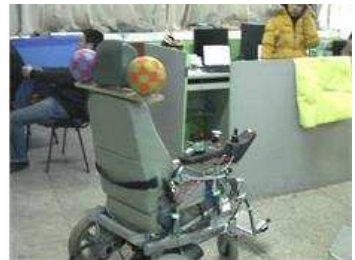

(3)

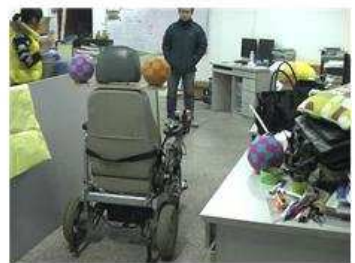

(6)

(a) Picture for first test of the fourth experiment

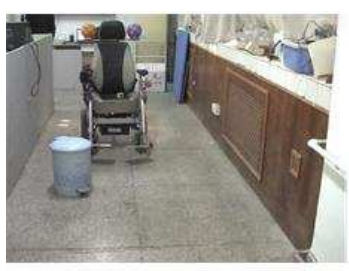

(1)

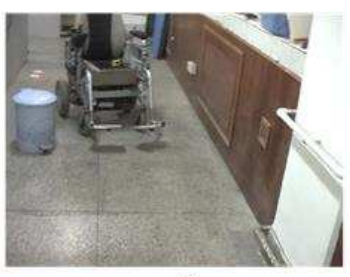

(2)

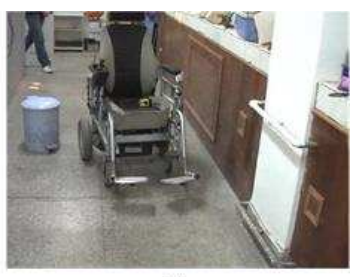

(3)

(b) Pictures for second test of the fourth experiment

Figure 9: Two experiments of harmonic navigation with collision-free 


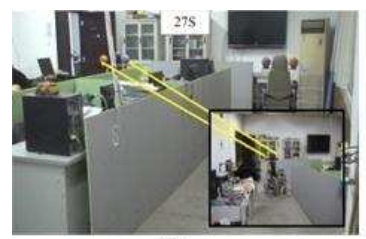

(1)

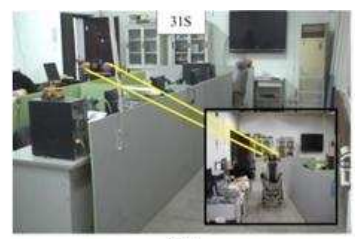

(2)

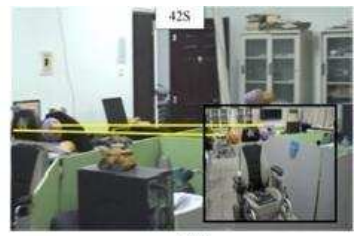

(3)

Figure 10: A series of snapshots of path finding involving a conflict between two wheelchairs (note that the yellow line indicate the location of the wheelchair in picture-in-picture) 